\section{A multicenter prospective study of first-line antibi- otic therapy for early-stage gastric mucosa-associ- ated lymphoid tissue lymphoma and diffuse large B-cell lymphoma with histological evidence of mucosa-associated lymphoid tissue}

Several independent clinical studies have demonstrated that first-line Helicobacter pylori (HP) eradication (HPE) therapy can lead to successful durable complete remission (CR) in $56-100 \%$ of patients with HP-positive, early-stage gastric extranodal marginal zone lymphoma of mucosa-associated lymphoid tissue (MALT lymphoma). ${ }^{1-4}$ According to the 2008 World Health Organization (WHO) Classification of Tumours of Haematopoietic and Lymphoid Tissues, diffuse large Bcell lymphoma (DLBCL) with accompanying MALT lymphoma should be classified as DLBCL, and not highgrade transformed MALT lymphoma, ${ }^{5,6}$ because gastric DLBCL are not necessarily transformed MALT lymphomas and cannot be distinguished from de novo DLBCL. ${ }^{5,6}$

Several prospective studies have demonstrated that first-line HPE results in CR in two-thirds of patients with HP-positive stage IE gastric DLBCL with histological evidence of MALT [DLBCL(MALT)], ${ }^{2,7}$ and other studies have also demonstrated that some patients with HP-positive gastric DLBCL(MALT) respond to HPE., ${ }^{2,8,9}$ These findings may also justify the expansion of the study population to include patients with stage IIE1 gastric DLBCL(MALT) in the present prospective trial of firstline HPE.

A $t(11 ; 18)(q 21 ; q 21)$ translocation results in the production of the fusion protein BIRC3-MALT1, causing constitutive activation of nuclear factor kappa-lightchain-enhancer of activated B cells (NF- $\mathrm{B}$ ) and contributing to the HP-independent growth of gastric MALT lymphomas. ${ }^{1,10}$ This translocation, however, rarely occurs in gastric DLBCL(MALT). ${ }^{2}$ Immunohistochemical detection of the nuclear translocation of $\mathrm{B}$-cell lymphoma/leukemia 10 (BCL10) or NF- $\mathrm{BB}$ is useful in predicting $\mathrm{HP}$ independence (the lack of complete lymphoma regression after HPE) in both early-stage MALT lymphoma and DLBCL(MALT) of the stomach, irrespective of the presence of the $t(11 ; 18)(q 21 ; q 21)$ translocation. ${ }^{1,2,11}$ In addition to HP-independent markers, we recently reported that the expression of cytotoxin-associated gene A (CagA) of the HP and CagA signaling molecules, phospho-Src homology-2 domain-containing phosphatase ( $\mathrm{p}$-SHP2) and phospho-extracellular signalregulated kinase (p-ERK), in tumor cells is closely associated with the HP dependence of gastric MALT lymphoma and gastric DLBCL(MALT). ${ }^{11,12}$

In April 2006, our institution and the National Health Research Institutes of Taiwan initiated a prospective phase II, multicenter trial evaluating the efficacy of firstline HPE treatment and the ability of nuclear BCL10 or NF- $\kappa \mathrm{B}$ expression and the $\mathrm{t}(11 ; 18)(\mathrm{q} 21 ; \mathrm{q} 21)$ translocation to predict HP independence in early-stage (i.e., stages IE and IIE1) HP-positive gastric MALT lymphoma and gastric DLBCL(MALT): the Taiwan Cooperative Oncology Group (TCOG) 3206 trial (ClinicalTrials.gov, NCT00327132). The enrolled patients were required to have histologically confirmed primary gastric lymphoma, including MALT lymphoma or DLBCL(MALT). ${ }^{5,6,13,14}$ Those who had undergone prior chemotherapy or radiotherapy for this tumor were excluded. MALT lymphoma and DLBCL(MALT) were diagnosed by histopathologists at individual hospitals, in accordance with specified criteria, with each diagnosis reviewed by members of the TCOG Pathology Committee. The diagnostic criteria for MALT lymphoma and DLBCL(MALT) are described in full in the Online Supplementary Methods). ${ }^{5,6,13,14}$ Detailed information on the staging work-up, diagnosis of HP infection, HPE regimens, and follow-up are provided in the Online Supplementary Methods and in Online Supplementary Figure S1. Full details on CR criteria [Groupe d'Etude des Lymphomes de l'Adult (GELA) histological scoring system] for tumors; ${ }^{15}$ molecular studies of the $t(11 ; 18)$ (q21;q21) translocation and immunohistochemical expression of BCL10, NF-кB, and CagA; primary and secondary endpoints; and statistical analyses are listed in the Online Supplementary Methods. All experimental protocols were approved by the Institutional Review Board of the Research Ethical Committee of National Taiwan University Hospital (n. 950606).

Of the 47 patients enrolled in this prospective study, one was excluded because of stage III disease. The clinicopathological characteristics of the 46 eligible patients, including 36 diagnosed with MALT lymphoma and 10 with DLBCL(MALT), are listed in Table 1 and in the Online Supplementary Results. The depth of lymphoma involvement in the gastric wall was determined by endoscopic ultrasonography in 28 patients. HP infection was successfully treated in all 36 patients with MALT lymphoma who completed the study protocol (22 after firstline treatment, 10 after second-line treatment and four after third-line treatment) ${ }^{16}$ and in nine $(90.0 \%)$ of the ten patients with DLBCL(MALT) after first-line treatment. Of the 46 patients, $34(73.9 \%)$ achieved complete remission (CR) (HP-dependent tumors) after the completion of HPE, including 26 of $36(72.2 \%)$ with MALT lymphoma and eight of the ten $(80 \%)$ with DLBCL(MALT) $(P=1.000)$ (Figure 1A-H). Of ten patients with DLBCL(MALT), three patients (diffuse blast components; $20-30 \%$ ) achieved CR, while five $(71.4 \%)$ of seven patients (diffuse blast components; 80-90\%) achieved CR. GELA criteria-based analyses showed that $30(88.2 \%)$ of the 34 patients who achieved CR did so within 12 months after the completion of HPE, whereas the other four $(11.8 \%)$ achieved CR after 12 months. The median time to CR after the completion of antibiotic therapy in all 34 patients was 4 months (range, 1-16 months), identical to that in patients with MALT lymphoma (4 months; range, 1-16 months) and DLBCL(MALT) (4 months; range, 1-10 months) $(P=0.530)$ (Figure 1I).

After a median follow-up of 87 months [95\% confidence interval (95\% CI): 75.2-98.8 months), only two patients died of causes unrelated to lymphoma - an 82year old woman with MALT lymphoma who died of cardiovascular disease and an 80-year old man with DLBCL(MALT) who died of pneumonia. No patient with MALT lymphoma who was unresponsive to HPE during treatment and follow-up showed high-grade transformation to DLBCL. The 8-year overall survival rate for all patients was $92.4 \%$ (95\% CI: $81.2-100 \%): 90 \%$ in patients with DLBCL(MALT) and $93.8 \%$ in patients with MALT lymphoma $(P=0.195)$ (Figure 1J). The median follow-up time after CR (calculated from the date of first CR to the date of tumor relapse or June 30,2018) was 80 months (95\% CI: 61.2-98.8 months) in all 34 HP-dependent patients, 82 months (95\% CI: 56.8-107.3 months) in the MALT lymphoma group, and 59 months (95\% CI: 23.0-95.0 months) in the DLBCL(MALT) group. Relapses were observed in two $(7.7 \%)$ of 26 patients with MALT lymphoma, but in none of the eight patients 
Table 1. Demographics and baseline characteristics of patients stratified by histological classification.

\begin{tabular}{|c|c|c|c|c|}
\hline Variables & $\begin{array}{c}\text { Total } \\
(n=46)\end{array}$ & $\begin{array}{l}\text { MALToma } \\
(n=36)\end{array}$ & $\begin{array}{c}\text { DLBCL(MALT) } \\
(n=10)\end{array}$ & $P$ \\
\hline Age & & & & 0.0049 \\
\hline Median (range), years & $53.5(29-85)$ & $51(29-79)$ & $71.5(48-85)$ & \\
\hline Gender, $\mathrm{n}(\%)$ & & & & 1.0000 \\
\hline Men & $17(37.0 \%)$ & $13(36.1 \%)$ & $4(40.0 \%)$ & \\
\hline Women & $29(63.0 \%)$ & $23(63.9 \%)$ & $6(60.0 \%)$ & \\
\hline Performance status (ECOG), n (\%) & & & & 1.0000 \\
\hline 0 & $25(54.4 \%)$ & $20(55.6 \%)$ & $5(50.0 \%)$ & \\
\hline 1 & $21(45.6 \%)$ & $16(44.4 \%)$ & $5(50.0 \%)$ & \\
\hline Symptom duration, n (\%) & & & & 0.3531 \\
\hline$<3$ months & $29(93.0 \%)$ & $20(55.6 \%)$ & $9(90.0 \%)$ & \\
\hline $3 \sim 12$ months & $9(19.6 \%)$ & $8(22.2 \%)$ & $1(10.0 \%)$ & \\
\hline$>12$ months & $4(8.7 \%)$ & $4(11.1 \%)$ & $0(0.0 \%)$ & \\
\hline None & $4(8.7 \%)$ & $4(11.1 \%)$ & $0(0.0 \%)$ & \\
\hline Lactate dehydrogenase, n (\%) & & & & 0.2015 \\
\hline Normal & $42(91.3 \%)$ & $34(94.4 \%)$ & $8(80.0 \%)$ & \\
\hline Abnormal & $4(8.7 \%)$ & $2(5.6 \%)$ & $2(20.0 \%)$ & \\
\hline$\beta_{2}$-microglobulin, n (\%) & & & & 0.0197 \\
\hline Normal & $33(71.8 \%)$ & $29(69.4 \%)$ & $4(40.0 \%)$ & \\
\hline Abnormal & $13(28.2 \%)$ & $7(19.4 \%)$ & $6(60.0 \%)$ & \\
\hline Clinical stage, n (\%) & & & & 0.5975 \\
\hline $\mathrm{IE}$ & $40(87.0 \%)$ & $32(88.9 \%)$ & $8(80.0 \%)$ & \\
\hline IIE-1 & $6(13.0 \%)$ & $4(11.1 \%)$ & $2(20.0 \%)$ & \\
\hline Tumor site, n (\%) & & & 0.6412 & \\
\hline Distant & $34(73.9 \%)$ & $25(69.4 \%)$ & $9(90.0 \%)$ & \\
\hline Proximal & $10(21.7 \%)$ & $9(25.0 \%)$ & $1(10.0 \%)$ & \\
\hline Mixed & $2(4.4 \%)$ & $2(5.6 \%)$ & $0(0.0 \%)$ & \\
\hline Endoscopic appearance, n (\%) & & & & 1.0000 \\
\hline Gastritis-like & $7(15.3 \%)$ & $6(16.6 \%)$ & $1(10.0 \%)$ & \\
\hline Infiltrative type with/without erosion & $3(6.5 \%)$ & $3(8.3 \%)$ & $0(0.0 \%)$ & \\
\hline Ulcerative type & $30(65.2 \%)$ & $23(63.9 \%)$ & $7(70.0 \%)$ & \\
\hline Polypoid type & $3(6.5 \%)$ & $2(5.6 \%)$ & $1(10.0 \%)$ & \\
\hline Mixed type & $3(6.5 \%)$ & $2(5.6 \%)$ & $1(10.0 \%)$ & \\
\hline EUS site ${ }^{* *}, \mathrm{n}(\%)$ & & & & 1.0000 \\
\hline Mucosa & $11(39.3 \%)$ & $9(37.5 \%)$ & $2(50.0 \%)$ & \\
\hline Submucosa & $12(42.9 \%)$ & $10(41.7 \%)$ & $2(50.0 \%)$ & \\
\hline Propria muscularis & $3(10.7 \%)$ & $3(12.5 \%)$ & $0(0.0 \%)$ & \\
\hline Whole-layers & $2(7.1 \%)$ & $2(8.3 \%)$ & $0(0.0 \%)$ & \\
\hline Tumor response, $\mathrm{n}(\%)$ & & & & 1.0000 \\
\hline $\mathrm{CR}$ & $34(73.9 \%)$ & $26(76.5 \%)$ & $8(80.0 \%)$ & \\
\hline Non-CR & $12(26.1 \%)$ & $8(23.5 \%)$ & $2(20.0 \%)$ & \\
\hline BCL10, n (\%) & & & & 1.0000 \\
\hline Cytoplasmic & $33(71.7 \%)$ & $26(72.2 \%)$ & $7(70.0 \%)$ & \\
\hline Nuclear & $13(28.3 \%)$ & $10(27.8 \%)$ & $3(30.0 \%)$ & \\
\hline NF-кB, n (\%) & & & & 0.7003 \\
\hline Cytoplasmic & $33(71.7 \%)$ & $25(69.4 \%)$ & $8(80.0 \%)$ & \\
\hline Nuclear & $13(28.3 \%)$ & $11(30.6 \%)$ & $2(20.0 \%)$ & \\
\hline CagA, n (\%) & & & & 0.2400 \\
\hline Negative & $13(28.3 \%)$ & $12(33.3 \%)$ & $1(10.0 \%)$ & \\
\hline Positive & $33(71.7 \%)$ & $24(67.7 \%)$ & $9(90.0 \%)$ & \\
\hline BIRC3-MALT1, n (\%) & & & & 0.5625 \\
\hline Negative & $42(91.3 \%)$ & $32(88.9 \%)$ & $10(100 \%)$ & \\
\hline Positive & $4(8.7 \%)$ & $4(11.1 \%)$ & $0(0.0 \%)$ & \\
\hline
\end{tabular}

N, number; MALToma, mucosa-associated lymphoid tissue lymphoma; DLBCL(MALT), diffuse large B-cell lymphoma with MALToma; ECOG, Eastern Cooperative Oncology Group; EUS, endoscopic ultrasonography; CR, complete remission. ${ }^{*} P$ values were calculated with the Fisher exact test for categorical variables, and with the nonparametric Wilcoxon rank sum test for continuous variables. **The depth of lymphoma involvement in the gastric wall was determined by endoscopic ultrasonography (EUS) in 28 patients. 
with DLBCL(MALT) who achieved CR $(P=0.419)$. Kaplan-Meier analysis showed that the 8-year relapsefree survival rates for HP-dependent cases were similar in the DLBCL(MALT) and MALT lymphoma groups (100\% vs. $92.3 \%, P=0.428$ ) (Figure $1 \mathrm{~K}$ ).

The clinicopathological features, response to HPE, expression of BCL10, NF- $\mathrm{KB}$ (p65), and CagA, and presence of $\mathrm{t}(11 ; 18)(\mathrm{q} 21 ; \mathrm{q} 21)$ translocation in the 36 patients with MALT lymphoma and ten patients with DLBCL(MALT) are summarized in Table 1 and in Online Supplementary Table S1. As shown in Figure 2, we found that the majority of CagA-positive cells (double immunohistochemical staining, brown color) were morphologically abnormal and expressed cluster of differentiation $(\mathrm{CD})^{20}$ (double immunohistochemical staining, green color) in HP-dependent cases of MALT lymphoma. In contrast, nuclear BCL10 and NF- $\mathrm{BB}$ (p65) expression was detected in tumor cells in HP-independent cases (Figure 2 and Online Supplementary Results). We also assessed serial changes in CagA expression levels in tumor cells before and after HPE treatment in four selected patients with HP-dependent gastric MALT lymphoma. As shown in Figure 2, expression of CagA in tumor cells was significantly downregulated in two patients (patients \#1 and \#2) who achieved partial remission and CR at 4 and 7 months, respectively, after completion of HPE. Gastric biopsies or remitting tumor cells of another two patients (patients \#3 and \#4) who achieved CR 1 and 4 months after completion of HPE showed no expression of CagA (Online Supplementary Figure S2).

We found that tumor location in the distal part of the stomach [hazard ratio $([\mathrm{HR})=4.667 ; 95 \%$ CI: $1.112-$ $19.589 ; P=0.035)$, absence of nuclear BCL10 expression (HR=51.667; 95\% CI: 7.530-354.519; $P<0.001)$, absence of nuclear NF- $\mathrm{KB}$ (p65) expression ( $\mathrm{HR}=22.50 ; 95 \% \mathrm{CI}$ : 4.227-119.768; $P<0.001)$, and CagA expression (HR=11.60; 95\% CI: 2.511-53.578; $P=0.002)$ were sig-
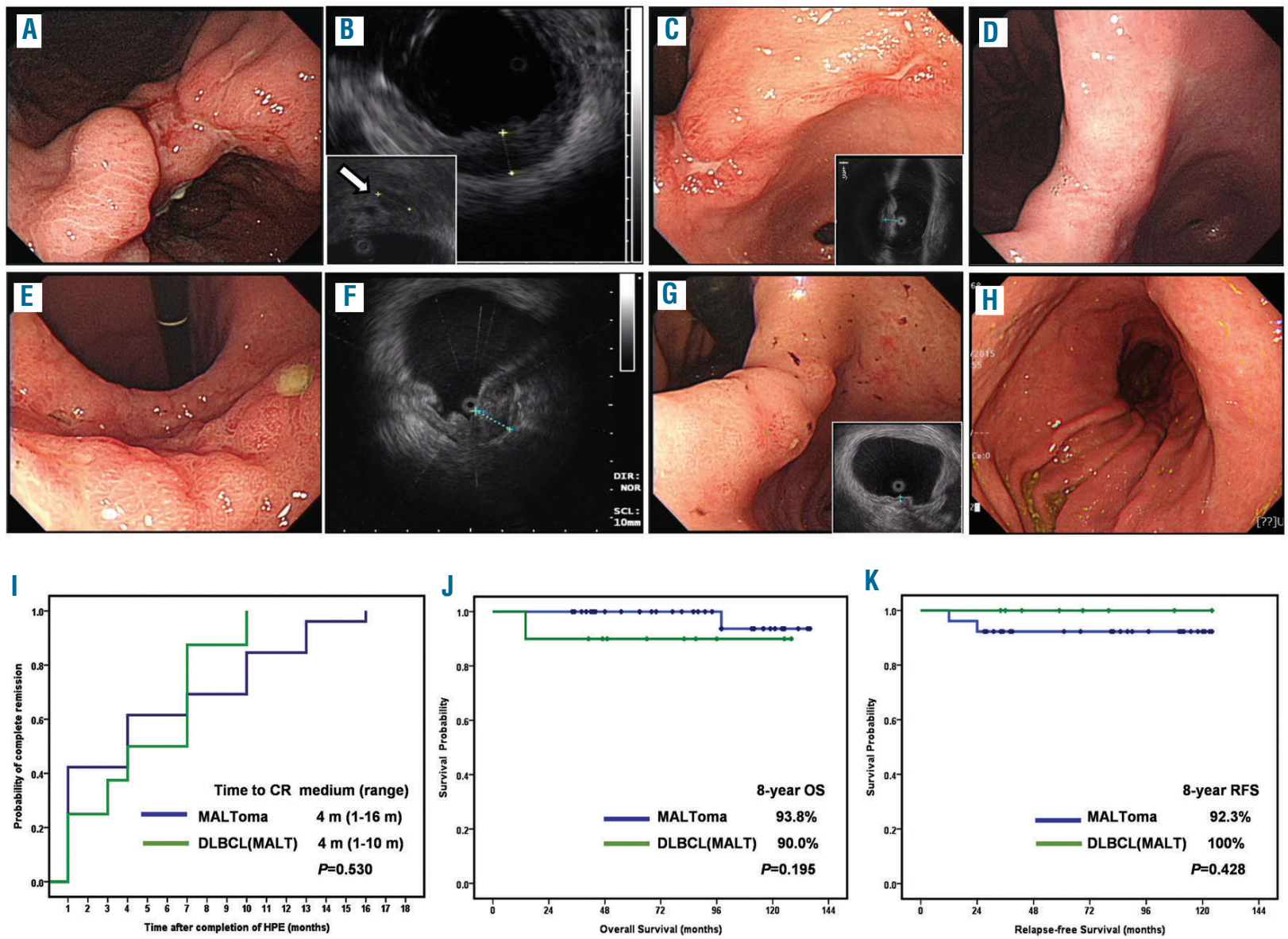

Figure 1. Changes of endoscopic features in cases of Helicobacter pylori-dependent gastric lymphomas treated with eradication therapy and time to complete remission and survival curves for patients. (A-H) Changes of endoscopic features in cases of Helicobacter pylori (HP)-dependent gastric diffuse large B-cell lymphoma [DLBCL mucosal-associated lymphoma tissue (MALT)] and MALT lymphoma before and after completion of HP eradication therapy (HPE). (A) Endoscopy showing an ulcerative mass in the angle in a 70-year old woman before HPE [histopathology disclosed DLBCL(MALT)]. (B) Endoscopic ultrasound (EUS) showing tumor with involvement of muscle layers (maximum thickness: $11 \mathrm{~mm}$ ) in the same case, A\#. Left bottom inset, hypoechoic lesions at the perigastric region (white arrow). (C) One month after completion of HPE, partial remission (PR) was achieved in the same case, A\#. Right bottom inset, muscle layer involvement (maximum thickness: $7.6 \mathrm{~mm}$ ) but no hypoechoic lesions at the perigastric region (D) Four months after completion of HPE, complete remission (CR) was achieved in the same case, A\# (E) Endoscopy showing huge hyperemic patches with nodularity at the angle and antrum and one $0.5 \mathrm{~cm}$ shallow ulcer at the angle in a 76-year old woman before HPE (histopathology disclosed MALT lymphoma). (F) EUS showing the tumor with whole layer involvement (maximum thickness: $12.6 \mathrm{~mm}$ ) in the same case, E\# (G) One month after completion of HPE, PR was achieved in the same case, E\#. Right bottom inset, whole layers with mild thickening $(2.3 \mathrm{~mm})(\mathrm{H})$ Seven months after completion of HPE, CR was achieved in the same case, E\#. (I-K) Time to complete remission and survival curves for patients who received HPE as first-line treatment. (I) Time to CR in 34 cases [26 with MALT lymphoma (MALToma) and 8 with DLBCL(MALT)] was calculated from the completion of antibiotic treatment to the first evidence of CR through Kaplan-Meier analysis. (J) The overall survival (OS) curve for all cases according to the histological subclassification. (K) The relapse-free survival (RFS) curve for HP-dependent cases according to the histological subclassification. 

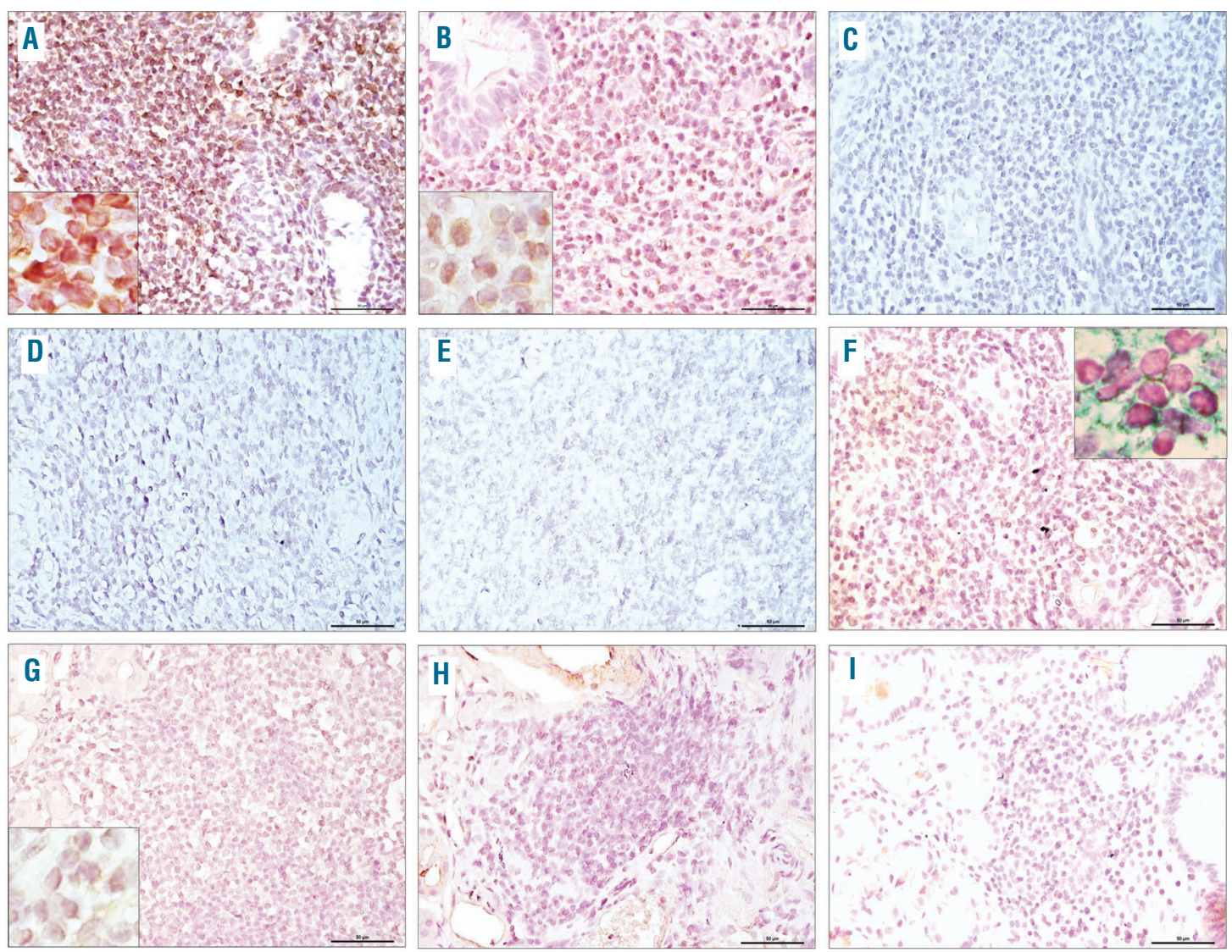

Figure 2. Examples of immunohistochemical analyses of BCL10, NF-kB, and CagA, and changes in the expression levels of CagA in serial samples of tumor cells before and after completion of Helicobacter pylori eradication therapy. (A) Nuclear BCL10 expression in tumor cells of a Helicobacter pylori (HP)-independent case (left bottom inset, $\times 1000$. (B) Nuclear NF-KB (p65) expression in tumor cells of a HP-independent case (left bottom inset, $\times 1000)$. (C) Negative expression of CagA in tumor cells of a HP-independent case. (D) Negative expression of BCL10 in tumor cells of a HP-dependent case. (E) Mild cytoplasmic expression of NF-KB (p65) in tumor cells of the HP-dependent case. (F) Expression of CagA in tumor cells of a HP-dependent case: double staining shows that tumor cells with CagA nuclear staining (brown color) are also CD20-positive (green color), right upper inset, $x$ 1000. (G) A high baseline expression of CagA in tumor cells of an HP-dependent case before HP eradication (HPE) (case \#1) (left bottom inset, $\times 1000$ ). (H) Decreased expression of CagA in remitting tumor cells (case \#1) 1 month after completion of HPE. (I) No expression of CagA in remitting tumor cells (case \#1) 4 months after completion of HPE (case \#1, the time to complete remission: 7 months).

nificantly associated with HP-dependent status. Multivariate analysis identified the absence of nuclear BCL10 expression (HR $=25.8747$; 95\% CI: 1.252534.653; $P=0.035)$ as an independent predictor of HP dependence for gastric MALT lymphoma and DLBCL(MALT). In contrast, distal lesion site ( $\mathrm{HR}=2.186$; 95\% CI: $0.173-27.570 ; P=0.545)$, absence of nuclear NF-кB (p65) expression ( $\mathrm{HR}=1.436$; 95\% CI: $0.076-$ 27.139; $P=0.809)$, and $\mathrm{CagA}$ expression $(\mathrm{HR}=2.063 ; 95 \%$ CI: 0.199-21.429; $P=0.544)$ were not associated with HP dependence in these tumors.

The current study showed that $16(61.5 \%)$ of the 26 patients with HP-dependent gastric MALT lymphoma achieved CR within 6 months, whereas six (23.1\%) required 6-12 months for $\mathrm{CR}$, and four (15.4\%) required 12-24 months after completing successful HPE. These findings are in agreement with previous reports showing that more than 12 months may be required for CR after completing HPE in patients with gastric MALT lymphoma (delayed responders). ${ }^{17,18}$ Consistent with findings in patients with gastric MALT lymphoma, the present study found that eight $(80.0 \%)$ of ten patients with gastric DLBCL(MALT) achieved CR at a median of 4.0 months after HP treatment had been completed.
Previous studies have demonstrated that patients with early-stage gastric DLBCL(MALT) who are unresponsive to antibiotics and subsequently treated with systemic chemotherapy achieve CR and remain disease-free during follow-up. 2,7,8,9 Although these results indicate that waiting for 6-7 weeks to observe the treatment response after initiating HPE does not affect clinical outcomes, it must be considered that patients with gastric DLBCL(MALT) who may experience progression during a lag of 6-7 weeks (first 2 weeks of antibiotic treatment) or initially respond to HPE may have a risk of tumor progression during follow-up if chemotherapy is delayed. Therefore, to avoid delaying chemotherapy for patients with gastric DLBCL(MALT) who receive first-line HPE, our current protocol recommends that endoscopic and imaging examinations should be arranged to assess tumor status if patients have predominant symptoms, and systemic chemotherapy should be prescribed immediately for those with stable or progressive disease.

Extended lymphoma invasion is closely associated with the HP independence of gastric MALT lymphoma. ${ }^{19}$ However, we found that depth of invasion was not closely associated with HP independence, as shown by the HP dependence in four patients with stage IIE1 
lesions, including two with DLBCL(MALT) whose tumors expressed CagA but lacked nuclear BCL10 and NF- $\kappa \mathrm{B}$ (p65) expression. However, the mechanisms underlying the regression of tumors in regional perigastric lymph nodes after HPE remain unclear, as these tumors are not in direct contact with HP and are usually composed of normal, not HP-specific, T cells. ${ }^{1,2,20}$

This study of first-line HPE in stage IE/IIE1 gastric MALT lymphoma and DLBCL(MALT) of the stomach also validated that our previously identified molecular markers, nuclear expression of BCL10 and NF-KB (p65), are closely associated with HP independence in these tumors, irrespective of the presence of the $\mathrm{t}(11 ; 18)(\mathrm{q} 21 ; \mathrm{q} 21)$ translocation. ${ }^{2,11}$ The current study also found that most HP-dependent tumors express CagA and lack nuclear BCL10 expression. Assessments of these HP-dependent tumors have shown that CagA expression in post-HPE gastric biopsy is downregulated, indicating that HP-associated CagA acts as a bacterial oncoprotein in stimulating the proliferation of $B$ cells, ${ }^{12,20,21}$ thereby contributing to the HP-dependent growth of these tumors.

In conclusion, the results of this prospective phase II trial demonstrate that first-line antibiotics, rather than 6 months of toxic systemic chemotherapies, cure a subset of gastric DLBCL(MALT) cases. Immunohistochemical assays for nuclear BCL10 or NF- $\mathrm{BB}$ (p65) expression and CagA expression can help predict HP dependence in patients with early-stage MALT lymphoma and DLBCL(MALT) of the stomach who receive first-line HPE. A "watch and wait"22 treatment strategy is appropriate for patients with gastric MALT lymphoma who have partial remission or stable disease and whose tumors do not express nuclear BCL10 or NF-kB (p65) 12 months after completing successful HPE, as a proportion will achieve subsequent CR (delayed responders). Further investigation of the molecular mechanisms underlying this delayed response is warranted.

Hui-Jen Tsai, ${ }^{1,2,3^{*}}$ John Jen Tai, ${ }^{1}$ Li-Tzong Chen, ${ }^{1,2,3^{*}}$ Ming-Shiang Wu, ${ }^{4}$ Kun-Huei Yeh, ${ }^{5,6,7}$ Chung-Wu Lin, ${ }^{8}$ Tsang-En Wang, ${ }^{9}$ Hsiu-Po Wang, ${ }^{4}$ Fang-Jung Yu, ${ }^{2}$ Jyh-Ming Liou, ${ }^{4}$ Chin-Fu Hsiao, ${ }^{1}$ Tsu-Yao Cheng, ${ }^{4}$ Hong-Jen Yeh, ${ }^{10}$ Chung-Wang Ko, ${ }^{10}$ Ming-Jen Chen, ${ }^{9}$ Gin-Ho Lo, ${ }^{11}$ Ping-I Hsu, ${ }^{11}$ Cheng-Shyong Chang, ${ }^{12}$ Wei-Shou Hwang, ${ }^{13}$ Shih-Sung Chuang, ${ }^{8,13,14}$ Hsiao-Wei Lee, ${ }^{5,6}$ Chia-Tung Shun, ${ }^{8}$ Chang-Fang Chiu, ${ }^{15}$ Wen-Ming Wang, ${ }^{2}$ Ching-Yun Hsieh, ${ }^{15}$ Tsang-Wu Liu, ${ }^{1}$ Jaw-Town Lin, $^{16}$ Sung-Hsin Kuo ${ }^{5,6,7}$ and Ann-Lii Cheng ${ }^{4,6,7}$ for the Taiwan Cooperative Oncology Group

*HJT and LTC contributed equally as co-first authors.

${ }^{1}$ National Institute of Cancer Research, National Health Research Institutes, Tainan; ${ }^{2}$ Department of Internal Medicine, Kaohsiung Medical University Hospital, Kaohsiung Medical University, Kaohsiung; ${ }^{3}$ Department of Internal Medicine, National Cheng-Kung University Hospital, Tainan; ${ }^{4}$ Department of Internal Medicine, National Taiwan University Hospital, Taipei; ${ }^{5}$ Department of Oncology, National Taiwan University Hospital, Taipei; ${ }^{6}$ Graduate Institute of Oncology, National Taiwan University College of Medicine, Taipei; 'Department of Medical Oncology, National Taiwan University Cancer Center, National Taiwan University College of Medicine, Taipei; ${ }^{8}$ Department of Pathology, National Taiwan University Hospital, Taipei; ${ }^{9}$ Division of Gastroenterology, Department of Internal Medicine, MacKay Memorial Hospital, Taipei; ${ }^{10}$ Department of Internal Medicine, Taichung Veterans General Hospital, Taichung; ${ }^{11}$ Department of Internal Medicine, Kaohsiung Veterans General Hospital, Kaohsiung; ${ }^{12}$ Department of Internal Medicine, Changhua
Christian Hospital, Changhua City; ${ }^{13}$ Department of Pathology, Chi-Mei Medical Center, Tainan; ${ }^{14}$ Department of Pathology, School of Medicine, Taipei Medical University, Taipei; ${ }^{15}$ Department of Internal Medicine, Graduate Institute of Clinical Medicine, China Medical University, Taichung and ${ }^{16}$ School of Medicine, Fu Jen Catholic University, New Taipei City, Taiwan

Presented in oral form at the $77^{\text {th }}$ Annual Meeting of the Japanese Society of Hematology, Osaka, Japan, 16-18 October, 2015.

Acknowledgments: the authors would like to thank the members of the Taiwan Cooperative Oncology Group (TCOG) (ShuChuan Lai, Bor-Rong Chen, and Cheng-Yu Chu) for statistical analyses, the process of enrolling patients and collecting their data and other necessary support.

Funding: this study was sponsored, conducted and analyzed by the TCOG and following research grants: MOST 107-2314-B002-217-MY3, MOST 107-2811-B-002-044-, and 108-2811B-002-616-from the Ministry of Science and Technology, Taiwan, and MOHW108-TDU-B-211-133002 from the Ministry of Health and Welfare, Taiwan.

Correspondence: SUNG-HSIN KUO - shkuo101@ntu.edu.tw doi:10.3324/haematol.2019.228775

Information on authorship, contributions, and financial \& other disclosures was provided by the authors and is available with the online version of this article at www. haematologica.org.

\section{References}

1. Du MQ, Isaccson PG. Gastric MALT lymphoma: from aetiology to treatment. Lancet Oncol. 2002;3(2):97-104.

2. Kuo SH, Cheng AL. Helicobacter pylori and mucosa-associated lymphoid tissue: what's new. Hematology Am Soc Hematol Educ Program. 2013;2013:109-117.

3. Zucca E, Bertoni F. The spectrum of MALT lymphoma at different sites: biological and therapeutic relevance. Blood. 2016;127 (17):2082-2092.

4. Raderer M, Kiesewetter B, Ferreri AJ. Clinicopathologic characteristics and treatment of marginal zone lymphoma of mucosa-associated lymphoid tissue (MALT lymphoma). CA Cancer J Clin. 2016;66(2):153-171.

5. Swerdlow SH, Campo E, Harris NL, et al. The World Health Organization Classification of Tumours of Haematopoietic and Lymphoid Tissues. Lyon, France: WHO; 2008.

6. Swerdlow SH, Campo E, Pileri SA, et al. The 2016 revision of the World Health Organization classification of lymphoid neoplasms. Blood. 2016;127(20):2375-2390.

7. Ferreri AJ, Govi S, Raderer M, et al. Helicobacter pylori eradication as exclusive treatment for limited-stage gastric diffuse large B-cell lymphoma: results of a multicenter phase 2 trial. Blood. 2012; 120(18):3858-3860.

8. Cavanna L, Pagani R, Seghini P, et al. High grade B-cell gastric lymphoma with complete pathologic remission after eradication of Helicobacter pylori infection: report of a case and review of the literature. World J Surg Oncol. 2008;6:35.

9. Kuo SH, Yeh $\mathrm{KH}, \mathrm{Wu} \mathrm{MS}$, et al. Helicobacter pylori eradication therapy is effective in the treatment of early-stage $\mathrm{H}$ pylori-positive gastric diffuse large B-cell lymphomas. Blood. 2012;119 (21):4838-4844.

10. Du MQ. MALT lymphoma: a paradigm of NF-кB dysregulation. Semin Cancer Biol. 2016;39:49-60.

11. Kuo SH, Chen LT, Lin CW, et al. Expressions of the CagA protein and CagA-signaling molecules predict Helicobacter pylori dependence of early-stage gastric DLBCL. Blood. 2017;129 (2):188-198.

12. Kuo SH, Yeh KH, Chen LT, et al. Helicobacter pylori CagA translocation is closely associated with the expression of CagAsignaling molecules in low-grade gastric mucosa-associated lymphoid tissue lymphoma. Am J Surg Pathol. 2015;39(6):761-766.

13. Isaacson PG, Du MQ. Gastrointestinal lymphoma: where morphology meets molecular biology. J Pathol. 2005;205(2):255-274.

14. Doglioni C, Ponzoni M, Ferreri AJ, et al. Gastric lymphoma: the histology report. Dig Liver Dis. 2011;43(Suppl 4):S310-318.

15. Copie-Bergman C, Wotherspoon AC, Capella C, et al. GELA histological scoring system for post-treatment biopsies of patients 
with gastric MALT lymphoma is feasible and reliable in routine practice. Br J Haematol. 2013;160(1):47-52.

16. Liou JM, Bair MJ, Chen CC, et al. Taiwan Gastrointestinal Disease and Helicobacter Consortium. Levofloxacin sequential therapy vs levofloxacin triple therapy in the second-line treatment of Helicobacter pylori: a randomized trial. Am J Gastroenterol. 2016;111(3):381-387.

17. Fischbach W. Goebeler ME, Ruskone-Fourmestraux A, et al. Most patients with minimal histological residuals of gastric MALT lymphoma after successful eradication of Helicobacter pylori can be managed safely by a watch and wait strategy: experience from a large international series. Gut. 2007;56(12):1685-1687.

18. Terai S, Iijima K, Kato K, et al. Long-term outcomes of gastric mucosa-associated lymphoid tissue lymphomas after Helicobacter pylori eradication therapy. Tohoku J Exp Med. 2008;214(1):79-87.
19. Zullo A, Hassan C, Cristofari F, et al. Effects of Helicobacter pylori eradication on early stage gastric mucosa-associated lymphoid tissue lymphoma. Clin Gastroenterol Hepatol. 2010;8(2):105-110.

20. Kuo SH, Wu MS, Yeh KH, et al. Novel insights of lymphomagenesis of Helicobacter pylori-dependent gastric mucosa-associated lymphoid tissue lymphoma. Cancers (Basel). 2019;11(4):547.

21. Paydas, $S$. Helicobacter pylori eradication in gastric diffuse large $B$ cell lymphoma. World J Gastroenterol. 2015;21(13):3773-3776.

22. Fischbach W, Dorlöchter C. Patients with gastric MALT lymphoma revealing persisting endoscopic abnormalities after successful eradication of Helicobacter pylori can be safely managed by a watch-and-wait strategy. Z Gastroenterol. 2019;57(5):593599. 